\title{
Die Bistumsblätter — universelle Medien der Ortskirche?
}

\author{
von Werner Döbereiner
}

„Die Kirchenzeitung hat die Pflicht - will sie ihrer Aufgabe, treu und redlich das Leben der Kirche widerzuspiegeln, gerecht werden - über alle wichtigen (und manchmal unwichtig scheinenden) Vorgänge in diesem lebendigen Leib der Kirche objektiv, ohne Beschönigung, nüchtern und sachlich $\mathrm{zu}$ informieren. ${ }^{\text {1 }}$

So umschreibt die Kirchenzeitung für das Bistum Limburg „Der Sonntag“ den Auftrag eines Bistumsblattes; in einer wissenschaftlichen Definition nimmt sich dies etwa so aus:

„Das Bistumsblatt ist ein gedrucktes Presse-Erzeugnis, dazu bestimmt, einer sachlich, nämlich durch das spezielle Interesse an den Geschehnissen (Ereignissen und Meinungen) in der Bistumskirche, der außer- und überdiözesanen Kirche sowie in Politik und Gesellschaft, welche die Kirche betreffen, räumlich - nämlich auf das jeweilige Bistum - und ideologisch — nämlich auf den katholischen Glauben - von vornherein begrenzten Empfängerschaft fortlaufend im wöchentlichen Abstand und vollständig das Wissen von jeweils höchstaktuellen, dieses spezielle Interesse berührenden Gegenständen auf unbegrenzte oder begrenzte Dauer zu vermitteln, wobei die Normen des Staates, der Gesellschaft und der Kirche (positiv oder negativ) berücksichtigt werden." 2

\section{Und die Wirklichkeit?}

Seit etwa einem Jahrzehnt wird im kirchlichen Raum verstärkt über die Vermittlungsansprüche und Vermittlungswirklichkeiten der Bistumsblätter diskutiert; so hat 1969 der Münchener Zeitungswissenschaftler Hans Wagner u. a. folgende Begründung für sein Modell eines „Kirchenzeitungsrates“ angeführt: „Der Leser will mit Hilfe seiner Kirchenzeitung die Meinungen und Vorstellungen z. B. seines Bischofs, verschiedener Gruppen und Persönlichkeiten usw. in der Bistumskirche erfahren." Als Aufgabe des Kirchenzeitungsrates ergibt sich hieraus, „darüber zu wachen, daß im Forum der Kirchenzeitung niemandem der Mund gestopft wird, daß keine wichtige Stimme übergangen wird, daß alle, die sich zu Wort melden und Vernünftiges zu sagen haben, auch wirklich zu Wort kommen “3. Fünf Jahre später prophezeite Hans Wagner gar das „Ende der katholischen Presse“4.

Diese zeitweise sehr hitzig geführte Diskussion stützte sich lediglich auf Vermutungen; exakte Daten darüber, ob die Bistumsblätter wirklich alle wichtigen Ereignisse und Meinungen vermitteln bzw. ob sie Wichtiges unterschlagen, konnte keiner der Kontrahenten vorlegen. Der Verfasser hat sich deshalb in seiner Dissertation ${ }^{5}$ die Aufgabe gestellt, dieses Datenmaterial - zumindest für Teilbereiche - beizubringen und damit einen Beitrag zu der Frage „Sind die Bistumsblätter universelle Medien der Ortskirche?“ zu leisten, ohne diese Frage endgültig lösen zu wollen oder zu können.

Werner Döbereiner ist Pressereferent in der Bayerischen Architektenkammer, München. Er promovierte zum Dr. phil. mit einer Arbeit über das Thema: „Die Bistumsblätter - universelle Medien der Ortskirche?" 
Ausgehend von den Anforderungen, welche die Zeitungswissenschaft an universelle Medien stellt (vgl. hierzu die Definition zu Beginn dieser Ausführungen) und den selbstgesetzten Vermittlungsansprüchen der Bistumsblätter wurde an vier Fallbeispielen (Enzyklika „Humanae vitae“, Zölibatsdiskussion, Motuproprio „Matrimonia mixta" und Vorbereitungsphase der Gemeinsamen Synode der Bistümer in der Bundesrepublik Deutschland) untersucht, inwieweit Universalität, d. h. Vollständigkeit der Ereignisse und Meinungen in den 22 Bistumsblättern ${ }^{\theta}$ gewährleistet ist. Dabei wurden vorwiegend folgende Aspekte betrachtet:

1. Werden alle Ereignisse und Meinungen vermittelt?

2. Kommen alle Personen und Gruppen in der Kirche zu Wort?

3. Kommen alle Personen und Gruppen aus dem jeweiligen Bistum zu Wort?

Es würde zu weit führen, hier zu erläutern, mit Hilfe welcher Methoden die Antworten auf diese Fragen gewonnen wurden; stattdessen sollen einige Ergebnisse diese Untersuchung beispielhaft herausgegriffen wenden.

\section{Zum Beispiel die Enzyklika "Humanae vitae"}

Am 25. Juli 1968 gab Papst Paul VI. die Enzyklika "Humanae vitae“ heraus, die unter den Stichworten "Geburtenregelung" oder vulgärer "Pille oder nicht" eine weltweite Diskussion auslöste. Diese von den verschiedensten Seiten geführte, argumentenreiche und lang andauernde Debatte mußte in den Bistumsblättern wiedergegeben werden. Deshalb wird die Inhaltsanalyse dieser Auseinandersetzung auch hier ausgewählt, da sie nicht nur über einen längeren Zeitraum hinweg, sondern auch von einer Vielzahl von Ausgangspartnern der unterschiedlichsten Anschauung und (geographischen und sozialen) Herkunft geführt wunde und - gerade durch die Verkürzung auf das Problem der Antibabypille - viele unmittelbar betraf, für viele also von größter persönlicher Aktualität war.

1. Die Enzyklika "Humanae vitae“ stand allen Bistumsblättern im vollen Wortlaut zur Verfügung; der gesamte Text wurde von elf Blättern wiedergegeben. Die Zielpartner, d. h. die Leser, konnten sich hier universell informieren und ihre Meinungsbildung auf der umfassenden Aussage des Papstes aufbauen. Nahezu vollständig wurde die Enzyklika von weiteren drei Bistumsblättern veröffentlicht; hierdurch entfallen u. U. wichtige Hinweise, die eine eigene Stellungnahme beeinflussen könnten. Die verbleibenden acht Untersuchungsobjekte vermitteln - größere oder kleinere Teile der Enzyklika nicht, so daß entweder bestimmte Gruppen oder die gesamte Zielpartnerschaft für sie ausschlaggebende, sie unmittelbar betreffende Informationen nicht erhalten und sich auch nicht (im positiven oder negativen Sinn) danach richten können. Auch wenden sie die gesamte nachfolgende Diskussion in einem anderen Licht sehen, da ihnen der zugrundeliegende Text nicht vollständig gegenwärtig ist. Anhand dieses Befundes ist somit festzustellen, daß eine Reihe von Bistumsblättern hinsichtlich der Vermittlung des Wortlautes der Enzyklika dem Anspruch, über alle wichtigen Ereignisse in der Kirche vollständig zu berichten, nicht gerecht wird`.

2. Zur Enzyklika "Humanae vitae" bringt die Katholische Nachrichten-Agentur (KNA) 1.410 Gesprächsdetails $^{8}$; auf diesem hier willkürlich angenommenen "Informations-Optimum“ basiert die Vermittlung aller 22 Bistumsblätter, weil es allen in gleicher Weise zur Verfügung steht. Allein aus räumlichen Gründen (jedes Blatt kann jeweils nur eine begrenzte Anzahl von Seiten füllen) ist es jedoch keinem Unter- 
suchungsobjekt möglich, alle von der KNA angebotenen Aussagen weiterzugeben. Der schon allein deshalb gebotene Selektionsvorgang führt zu einer Vermittlung von 191 Gesprächsdetails im „schlechtesten “ und 528 Gesprächsdetails im „besten“ Fall. Je mehr Aussagen vermittelt werden, umso vollständiger ist das Bild, das sich der einzelne machen kann, umso eher besteht die Möglichkeit, daß auch die Ansicht des einzelnen Lesers wiedergegeben wird, daß sich der einzelne in das Meinungsspektrum einordnen, seinen Standort in der Kirche bestimmen kann.

3. Je ausgeprägter das Selektionsverhalten des Vermittlers, d. h. der Redaktion, ist, je weniger Gesprächsdetails also vermittelt werden, desto größer ist die Gefahr, daß die Auswahl einseitig vorgenommen wird, daß das bestehende Meinungsbild (so wie es sich im KNA-Angebot widerspiegelt) in diese oder jene Richtung verzerrt wird. Bei KNA überwiegen mit $26 \%$ die ablehnenden Aussagen zur Enzyklika; dieser Schwerpunkt wird nur von einem Bistumsblatt übernommen. Nur die Leser dieses Blattes können ihre eigene Meinungsbildung auch an der tatsächlichen Meinungsverteilung orientieren. Bei allen übrigen Bistumsblättern ist die Verteilung der von der Katholischen Nachrichten-Agentur übernommenen Meinungen nach einer oder nach mehreren Richtungen verschoben. Jede Über- oder Unterbewertung einer Richtung hat jedoch eine Verzeichnung des Meinungsbildes zur Folge, die es dem Leser unmöglich macht, sich über die tatsächliche Meinungsverteilung $\mathrm{zu}$ informieren und sich gegebenenfalls selbst in das Meinungsgefüge einzuordnen. Mit jeder Verzeichnung des Meinungsbildes schwindet auch der Anspruch, universell zu informieren.

Besonders hingewiesen sei darauf, daß häufig die negative Richtung, also die Ablehnung der Enzyklika, zu kurz kommt; zahlreiche Vermittler gaukeln ihren Lesern vor, die Opposition gegen die Enzyklika sei ja gar nicht so groß gewesen. Sie machen damit die Zielpartner unsicher und unterschlagen möglicherweise gerade die Meinung des einzelnen Lesers, der sich dann in seinem Bistumsblatt nicht repräsentiert sieht, sich nicht in das gesamte Meinungsgefüge einordnen kann, plötzlich nicht in die Gemeinschaft des Dialogs eingebunden, sondern mit seiner Meinung isoliert ist.

4. Von der Redaktion verfaßte Beiträge zum Thema "Enzyklika“ zeigen, in welchem Umfang sich die Vermittler bemühen, durch inhaltlich und gestaltungsmäßig besonders auf die jeweiligen Leser abgestimmte Aussagen speziellen Interessen Rechnung zu tragen, die im Angebot der KNA nicht oder nicht ausreichend erfaßt sind, seien es besondere Informationen, seien es besondere, im jeweiligen Bistum angesehene und bekannte Persönlichkeiten, deren Meinung ein großes Gewicht besitzt. Nicht zuletzt zeigen diese Beiträge auch, inwieweit ein Bistumsblatt dem Anspruch, alle Meinungen zu Wort kommen zu lassen, dadurch gerecht $\mathrm{zu}$ werden versucht, daß sie Meinungen und Gesprächspartner in den Dialog hereinholt, die von der KNA nicht oder für den speziellen Fall nicht genügend einbezogen worden sind.

Bei den einzelnen Bistumsblättern umfassen diese redaktionellen Beiträge in keinem einzigen Fall bis zu 256 Gesprächsdetails.

War in der Auswahl der von KNA übernommenen Aussagen die Haltung der einzelnen Redaktionen (oder der Herausgeber) erkennbar, so läßt sich dies bei den redaktionellen Beiträgen nicht unbedingt feststellen; auffallend ist nämlich, daß sich hier nur wenige Bistumsblätter in einer bestimmten Richtung (positiv oder negativ eingestellt) festlegen, während die Mehrzahl auch in den redaktionellen Teilen auf unverbindliche, neutrale oder fordernde („man müßte“, "man sollte") Aussagen ausweicht, die keine eindeutige Stellungnahme erkennen lassen. Der Zielpartner, der 
Leser also, der für seine Meinungsbildung Hilfe von der Redaktion, die mit dem Problem und mit den Auffassungen in der Diözese vertraut ist, erwartet, wird im Stich gelassen und mit unverbindlichen Informationen „abgespeist “. So kommen zwar in den redaktionellen Beiträgen zusätzlich zu den von KNA vermittelten Aussagen Ausgangspartner zu Wort, aber häufig äußern sie keine klare Meinung; deshalb ist zu überlegen, ob durch die Vermittlung solcher Aussagen der Anspruch erfüllt werden kann, alle Meinungen (!) zu Wort kommen zu lassen.

5. Die Anzahl und der jeweilige Umfang der Leserbriefe gibt Aufschluß über das Bestreben der Redaktionen, vorwiegend Gesprächspartner aus dem Bistum, die nicht in die "Hierarchie " eingebunden sind und damit die Möglichkeit haben, sich ex officio zu äußern, in den Dialog einzubeziehen. Hier reicht die Skala der Gesprächsdetails von 0 bis 256, hier zeigt sich am deutlichsten, inwieweit Wert auf die Vollzähligkeit, die Universalität der Meinungen gelegt wird, inwieweit auch der Anspruch verwirklicht wenden soll, Forum des Dialogs der Bistumsgemeinde, universelles Medium der Ortskirche zu sein.

Bemerkenswert ist die große Zahl der Bistumsblätter, die in der Auswahl der Leserbriefe einen negativen Schwerpunkt setzen und damit der sich im KNA-Angebot widerspiegelnden Meinungsverteilung nahekommen.

6. Weitere Hinweise auf die Erfüllung des Anspruchs, umfassend zu informieren, liefert eine Auflistung der jeweils zu Wort kommenden Personen und Gruppen (Ausgangspartner); denn nur die Vollständigkeit der Ausgangspartner gewährleistet letztlich, daß alle Meinungen berücksichtigt und die tatsächlichen Meinungsverteilungen wiedergegeben werden. Um dies zu überprüfen, wurde ein Schema der möglichen Ausgangspartnergruppen entwickelt und jeder Ausgangspartner, der auftrat, einer dieser Gruppen zugeordnet.

In allen Bistumsblättern kamen folgende Ausgangspartner zu Wort:

- Papst

- Konzil

- Bischöfe

- wissenschaftliche Theologen

- Andersgläubige

- katholische Verbände

- sonstige identifizierbare Ausgangspartner, d.h. namentlich genannte Einzelpersonen, die keiner anderen Gruppe zugeordnet werden können

- sonstige anonyme Ausgangspartner, d.h. namentlich nicht genannte Einzelpersonen, z. B. „S.W. in T." oder „eine Mutter von drei Kindern", bzw. nicht näher einzuordnende informelle Gruppen, z. B. „kritische Stimmen“ oder die sprichwörtlichen "gut unterrichteten Kreise".

Von den beiden zuletzt genannten abgesehen, handelt es sich bei den Ausgangspartnern, die in allen Bistumsblättern präsent sind, um bekannte und populäre Gesprächspartner, die als Autoritäten gelten, deren Meinung großes Gewicht hat. Alle übrigen, hier nicht aufgeführten Gesprächspartner werden mehr oder weniger häufig vermittelt. Ein einziges Bistumsblatt läßt nahezu alle Ausgangspartnergruppen zu Wort kommen, kann also in dieser Beziehung den Anspruch erheben, einen umfassenden 
Dialog wiederzugeben. Von der Verwirklichung dieses Anspruchs entfernen sich mit zunehmender Zahl der nicht vermittelten Ausgangspartner alle übrigen Bistumsblätter. In einem Blatt beispielsweise kommen nur noch zwölf der insgesamt zweiunddreißig Gruppen zu Wort, der Leser weiß überhaupt nicht mehr, welche Personen und Gruppierungen sich zu Wort gemeldet haben, der Dialog dürfte äußerst unvollständig sein, der Anspruch, Organ des Dialogs in der Kirche zu sein, wird nur sehr unzureichend erfüllt.

7. Da das Bistumsblatt insbesondere den Anspruch erhebt, Organ der Ortskirche zu sein (dadurch unterscheidet es sich auch von der sonstigen kirchlichen Presse), sollte auch festgestellt werden, welche der jeweiligen Diözese angehörenden Ausgangspartner nicht zu Wort kommen, da anzunehmen ist, daß gerade sie aus der örtlichen Kenntnis heraus eine den speziellen Kommunikationsbedürfnissen gerecht werdende Stellungnahme abgeben können und ihren Aussagen aufgrund der Zugehörigkeit zum Bistum, vielleicht auch aufgrund persönlicher Bekanntschaft („einer von uns"!) besonderes Gewicht zukommt.

Die Untersuchung erbrachte u. a., daß in sieben Bistumsblättern nicht einmal die Diözesanbischöfe sich zur Enzyklika äußern; die nachkonziliaren Räte, die ein Bindeglied zwischen der Bistumshierarchie und dem „Bistumsvolk“ darstellen sollen, kommen gar in siebzehn Blättern nicht zu Wort.

Bereits diese beiden Beispiele zeigen, daß ein Dialog im Bistum ohne Gesprächspartner aus dem Bistum nahezu unmöglich ist. Der Anspruch, Organ der Bistumskirche zu sein, ist dann nicht erfüllt; denn im Organ der Bistumskirche sollten gerade die Gesprächspartner aus dem Bistum ihre Meinungen kundtun und so in den Dialog mit ihrer nächsten Umgebung eintreten.

Die Redaktionen als Anwälte dieses Gesprächs haben die Pflicht, für einen umfassenden Dialog zu sorgen - umfassend hinsichtlich der angebotenen Meinungen und umfassend hinsichtlich der zu Wort kommenden Gesprächspartner. Gesprächspartner, die sich nicht von sich aus zu Wort melden, sind um eine Aussage zu bitten, notfalls sind sie zu einer Stellungnahme zu provozieren. Nur so kann das Bistumsblatt dem Anspruch, universelles Medium der Ortskirche zu sein, gerecht werden.

\section{Also doch kein universelles Medium?}

Am Ende dieser Ausführung kann keine "Preisverleihung" stehen — schon allein deshalb nicht, weil keine Namen genannt und weil nur einige Ergebnisse zu einem von vier Fallbeispielen exemplarisch herausgegriffen wurden. Auch wenn diese Einschränkungen wegfielen, könnte keine „Preisverleihung“ stattfinden; denn das universelle Bistumsblatt gibt es nicht. Die Untersuchung hat vielmehr deutlich gemacht, daß die Ansprüche auf Universalität vom gleichen Bistumsblatt in verschiedenen Bereichen sehr unterschiedlich erfüllt werden.

Kehren wir zu den drei eingangs gestellten Fragen zurück, so lassen sich - jeweils auf einen Satz gebracht - folgende Antworten finden:

1. Kein Bistumsblatt erfüllt den Anspruch, alle Ereignisse und Meinungen in der Kirche zu vermitteln; d. h. eine Universalität der Vermittlung besteht bei keinem Bistumsblatt.

2. Kein Bistumsblatt läßt alle Personen und Gruppen in der Kirche zu Wort kommen; d.h. das Dialogforum ist bei keinem Untersuchungsobjekt vollständig. Da 
jedoch nur ein vollständiges Dialogforum die Gewähr für ein umfassendes Meinungsspektrum bietet, kann von einem unvollständigen Dialogforum auf eine nicht umfassende Wiedergabe der Meinungen geschlossen werden.

3. Kein Bistumsblatt läßt alle Personen und Gruppen im Bistum zu Wort kommen; d. h. das diözesane Dialogforum ist bei keinem Bistumsblatt vollständig, nicht alle Meinungen aus dem Bistum werden in den Dialog einbezogen.

Besonders beim Dialog im Bistum sind, wie gezeigt wurde, große Lücken festzustellen. Diesem ureigensten Bereich, der erst den Anspruch des „Lokalzeitungscharakters" rechtfertigt, müssen künftig die Redaktionen und Herausgeber mehr Beachtung schenken.

Abschließend sei davor gewarnt, die hier dargestellten Ergebnisse, so allgemein sie auch klingen, $\mathrm{zu}$ verallgemeinern; es wurde mehrfach darauf hingewiesen, daß sie im Gegenteil sehr differenziert zu sehen sind und nur für den jeweiligen Bereich Guiltigkeit besitzen. Wenn es auch das universelle Medium der Ortskirche nicht gibt, so gibt es doch Bistumsblätter, die in Teilbereichen der Verwirklichung dieses Anspruchs nahekommen - und dies ist bereits mehr, als manche Kritiker der Bistumsblätter wahrhaben wollen.

Anmerkungen:

1. „Der Sonntag. Kirchenzeitung für das Bistum Limburg“, Heft 3/1969.

2. Döbereiner, Werner: Die Bistumsblätter - universelle Medien der Ortskirche? Phil. Diss. München 1977, S. 47 f.

3. Wagner, Hans: Kirchenzeitungsrat in der Diskussion, in: „St. Willibaldsbote. Kirchenzeitung für das Bistum Eichstätt", Heft 12/1969.

4. So der Titel einer dreibändigen Schrift, erschienen Aschaffenburg 1974.

5. Vgl. Anm. 2.

6. Aus Gründen der wissenschaftlichen Abgrenzung wird in diesem Beitrag von „Bistumsblättern" und nicht von "Kirchenzeitungen " gesprochen; denn eine Zeitung verlangt die Vollständigkeit der Ereignisse aus allen Bereichen, während die Inhalte eines Blattes von vornherein sachlich begrenzt sind; vgl. hierzu Groth, Otto: Die unerkannte Kulturmacht. Bd. 1. Berlin 1960, S. 621.

7. Auf die Nennung einzelner Bistumsblätter wird bewußt verzichtet, da es hier nicht darum geht, "Zensuren ${ }^{\circ} \mathrm{zu}$ verteilen, sondern allgemeine Tendenzen aufzuzeigen und daraus Folgerungen zu ziehen.

8. Eine ausführliche Definition dieser für die Untersuchung als Hilfskonstruktion geschaffenen kleinsten zählbaren Einheit einer Aussage kann im Rahmen dieses Beitrags nicht gegeben werden.

\section{S UM M A R Y}

This contribution deals with the question of how far the diocesan weeklies in the Federal Republic of Germany can be seen as the universal media of the local Church. Three questions are posed: (1) Is all news and every opinion reported? (2) Are all members and groups within the Church free to express their opinion? (3) Has every group in each diocese 
this opportunity? According to the survey of concrete examples, the author comes to the conclusion that this is not so. He warns, however, against generalising from these results. In part, some of the weeklies come near to fulfilling these expectations - a fact which some critics would prefer to ignore.

\section{RESUME}

L'auteur présente, dans cet article, le compte-rendu du résultat de sa dissertation dans laquelle il examina la question suivante: à quel point les feuilles épiscopales, en République Fédérale d'Allemagne, sont-elles les moyens universels de l'église locale? Il examina les 22 feuilles épiscopales disponibles sur trois questions: 1. Tous les évènements et toutes les opinions sont-ils procurés? 2. Toutes les personnes et tous les groupes de l'église ont-ils la parole? 3. Toutes les personnes et tous les groupes de l'évêché en question ont-ils la parole? A l'aide d'interprétation pratique, il parvient à un résultat négatif, pour chacune des trois questions. Cependant il met en garde contre la généralisation de ce résultat. Car, dans certaines fractions de domaines, certaines feuilles épiscopales sont proches de la réalisation de la revendication, ce que certains critiques de feuilles épiscopales ne veulent pas admettre.

\section{R E S U M E N}

El autor presenta en este trabajo, resumida, la conclusión de su tesis, en la que estudió la medida en que las publicaciones diocesanas en la República Federal de Alemania son medios de comunicación universal en la iglesia local. Hizó una encuestra entre 22 semanarios diocesanos con estas tres preguntas: 1. Se publican todos los acontecimientos y opiniones? 2. Se da la palabra a todas las personas y grupos de la Iglesia, 3. Se da la palabra a todas las personas y grupos de la diócesis? En las tres cuestiones llega a una respuesta negativa. Advierte, sin embargo, del peligro de generalizar el resultado, pues diversos órganos diocesanos se aproximan a la realización del ideal, hecho que no quieren reconocer algunos críticos de la prensa diocesana. 